\title{
地球磁层-电离层系统对超强太阳风暴响应的数值 模拟研究
}

\author{
王赤 ${ }^{(1)}$, 李晖 ${ }^{(1)}$, 郭孝城 ${ }^{(1)}, 丁^{\left(U^{2}\right.}{ }^{(1)}$, 黄朝晖 ${ }^{(1)}$ \\ (1) 中国科学院空间科学与应用研究中心空间天气学国家重点实验室, 北京 100190; \\ (2) 中国科学院研究生院, 北京 100049 \\ *E-mail: cw@spaceweather.ac.cn
}

收稿日期: 2011-03-03; 接受日期: 2012-02-15

国家自然科学基金(批准号: 40921063, 40974106, 40831060)和科技部国家重点实验室专项经费资助

\begin{abstract}
摘要随着第 24 周太阳活动峰年(2012 2014 年)的临近, 超强太阳风暴可能导致的地球 空间环境的变化日益受到人们的关注. 本文以 1859 年发生的卡林顿事件的地磁观测数据为 出发点, 估计当时的行星际空间太阳风条件, 并利用全球三维磁流体力学模拟研究分析地 球磁层-电离层系统对超强太阳风暴的响应. 主要的结论有: (1) 向阳侧磁层顶和马激波被 压缩至 4.3 和 $6.0 \mathrm{Re}$ (地球半径), 同时侧翼部分的弓激波和磁层顶也受到强烈压缩作用. 磁 层顶压缩到地球同步轨道内是普遍现象, 地球同步轨道卫星直接暴露在磁鞘太阳风中. (2) 超强磁暴发生后, 1 区场向电流急剧增强约 60 倍, 电离层的越极电位降增加约 80 倍, 重联 电压为一般磁暴时期的 5 6 倍, 大量的太阳风能量进入到磁层内部, 从而引发强烈的空间 天气现象.
\end{abstract}

关键词

空间天气

太阳风暴

磁层-电离层系统

数值模拟
太阳活动会导致地球空间环境的剧烈变化, 从 而影响航天、通信、导航、定位和电力等天基和地基 高技术系统. 随着第 24 周太阳活动峰年(2012 2014 年)的临近, 超强太阳风暴可能导致的地球空间环境 的变化日益受到人们的关注. 人类历史上第一次有 观测记录的太阳耀斑发生在 1859 年 9 月 1 日. 两个 英国的天文学家记载了这次耀斑爆发的过程, 卡林 顿(Lord R.C. Carrington)就是其中之一. 事实表明, Carrington 观测的白光耀斑仅仅是一系列大规模太阳 活动以及对地效应的一部分. 后来, 人们把这次太阳 耀斑爆发以及随后发生的剧烈地球空间扰动称为 “Carrington Event”(卡林顿事件), 这是第一次也是最
强的一次由太阳剧烈活动引起地球空间扰动的空间 天气事件. 在此超级磁暴期间, 地磁 Dst 指数下降至 低于-1500 nT, 并在较低纬度上形成极光. Dst 指数 是地磁赤道附近 4 个均匀分布在不同经度上的地磁 台站的地磁水平强度变化的小时平均值, 一般磁暴 的 Dst 变化在-200 nT 以内. 卡林顿空间天气事件严 重影响了当时的电报业务, 全球有 $200000 \mathrm{~km}$ 的电报 线路受到严重损害, 很多线路的通讯中断 8 小时或更 长时间, 造成了较大的社会经济影响 ${ }^{[13]}$. 然而, 1859 年人类还没有进入空间时代, 对空间的依赖程度远 没有当今社会如此强烈. 有学者预测, 如果现在发生 类似强度的事件将造成约 700 亿美元的经济损失 ${ }^{[4]}$.

英文引用格式: Wang C, Li H, Guo X C, et al. Numerical study on the response of the Earth's magnetosphere-ionosphere system to a super solar storm. Sci China Earth Sci, 2012, doi: 10.1007/s11430-012-4405-4 
地球的磁层-电离层系统是地球空间环境的重要 组成部分, 地球弓激波和磁层顶的位置和形状一直 是人们关心的地球磁层大尺度结构. 根据卫星观测 数据, 人们给出了有关弓激波和磁层顶的经验公 式 ${ }^{[5,6]}$. 然而, 这些经验公式是根据卫星穿越弓激波 和磁层顶事件的统计分析而得到的, 其中在大磁暴 条件下的样本很少, 对大磁暴条件下的弓激波和磁 层顶的描述基本不具备统计意义. Shue 等 ${ }^{[7]}$ 描述了作 为太阳风动压和行星际磁场 (IMF) 南向分量 $\left(B_{z}\right)$ 函数 的磁层顶位置和形状. 由于 IMF $B_{z}$ 低于 $-20 \mathrm{nT}$ 的事 件非常少, 他们不能给出比较合理的数据拟合曲线, Shue 的磁层顶经验模型在 IMF $B_{z}<-20 \mathrm{nT}$ 就失效了. 而卡林顿事件的 IMF $B_{z}$ 据估计到达 $-60 \mathrm{nT}$ 以下, 有 关磁层顶和弓激波位置和形状的经验公式不再适用. 另外一方面, 由于卫星轨道的限制, 目前弓激波和磁 层顶的穿越大多都集中在向阳面低纬地区，对高纬 地区的弓激波和磁层顶缺乏观测. 除了磁层大尺度 结构之外, 地球磁层-电离层的大尺度电流体系是空 间环境要素磁场的控制因素, 在极端行星际条件下 表现出截然不同的性质. 例如, 在一般行星际条件下 (如太阳风速度为 $400 \mathrm{~km} / \mathrm{s}$, 行星际磁场(IMF) 为零、 5 $\mathrm{nT}$ 正北或 $5 \mathrm{nT}$ 正南的情况下), 1 区场向电流通过磁 层顶电流闭合，磁场顶作为 1 区场向电流的“供电源”. 然而, 最近的模拟结果表明, 在强 IMF 南向的磁暴条 件下 (如 IMF $B_{z}=-20 \mathrm{nT}$ ), 1 区场向电流增加, 可是磁 层顶电流反而减弱, 此时弓激波将给 1 区场向电流供 电, 为太阳风直接影响电离层提供通道 ${ }^{[8]}$. 因而, 利 用数值模拟的方法来分析极端行星际条件下的地球 磁层-电离层系统是目前最为有效的研究手段. Ridley 等 ${ }^{[9]}$ 利用密执根大学的全球三维磁层磁流体力学模 型(BATSRUS) $)^{[10]}$ 研究了磁层和电离层对类似卡灵顿 事件的超强行星际激波和日冕物质抛射事件的响应, 主要关注扰动在地球磁层-电离层系统的传播和相互 作用过程, 没有定量给出地球磁层大尺度结构、场向 电流和电离层越极电位降等近地空间环境要素的变 化. 本文将以 1859 年发生的卡林顿事件的地磁观测 数据为出发点, 估计行星际空间太阳风条件, 并利用 我们自主研发的全球三维磁流体力学模拟研究分析 地球磁层-电离层系统对超强太阳风暴的响应, 定量 给出的地球磁层大尺度结构、1 区场向电流和电离层 的越极电位降等空间环境要素的变化值, 增进人们 对极端灾害空间天气事件后果的定量认识.

\section{1 卡林顿事件的行星际条件估计}

卡林顿事件发生的年代还没有卫星观测，没有 行星际太阳风的观测数据, 但地磁观测的数据有很 长的历史. 我们可以根据地磁观测, 通过一定的理论 假设来估计行星际条件.

根据印度孟买地磁台的历史记录，1859 年 9 月 1 2 日发生的卡林顿事件期间地磁 Dst 指数开始上升 到达峰值 $120 \mathrm{nT}$, 然后下降至低于 $-1500 \mathrm{nT}^{[11]}$. $\mathrm{Li}$ 等 ${ }^{[12]}$ 利用改进的 Temerin 和 Li 的 Dst 预测模型 ${ }^{[13]}$, 为了再现卡林顿事件的 Dst 的变化, 推算激波到达后 的行星际太阳风的密度达到 $40 \mathrm{~cm}^{-3}$, 而后急剧增加 到 $1800 \mathrm{~cm}^{-3}$, 太阳风速度先迅速增加至 $1200 \mathrm{~km} \mathrm{~s}^{-1}$, 而后缓慢增加至 $1750 \mathrm{~km} \mathrm{~s}^{-1}$, 在此期间行星际南向 磁场达到-68nT. Wang 等 ${ }^{[14]}$ 利用地磁观测数据, 估 计了行星际激波动压平方根的变化. 根据对行星际 激波与地磁 $D s t$ 指数的统计分析结果, 行星际激波上 下游太阳风动压平方根的变化与磁暴急始的地磁指 数 SYM-H 变化有如下经验公式: $\Delta \sqrt{P_{d}}=$ $0.056 \times \Delta \mathrm{SYM}-H\left(\mathrm{nPa}^{1 / 2}\right)$, 可以估算出行星际激波太 阳风动压平方根变化约为 $6.7 \mathrm{nPa}^{1 / 2}$, 与利用 $\mathrm{Li}$ 等 ${ }^{[12]}$ 估算的太阳风参数计算的结果基本一致. 参考以上 估算, 我们在计算中选取以下太阳风参数来模拟卡 林顿事件的行星际条件(图 1): (1) 初始太阳风条件: $B_{z}=2 \mathrm{nT}, N=5 \mathrm{~cm}^{-3}, V_{x}=-450 \mathrm{~km} \mathrm{~s}^{-1}, T=0.91 \times 10^{5} \mathrm{~K}$; (2) 暴时太阳风条件: $B_{z}=-60 \mathrm{nT}, N=40 \mathrm{~cm}^{-3}, V_{x}=-1500$ $\mathrm{km} \mathrm{s}^{-1}, T=14 \times 10^{5} \mathrm{~K}$.

\section{2 数值模拟结果}

我们利用自主研发的三维全球磁层 MHD 模型 PPMLR-MHD ${ }^{[15]}$ 来模拟地球磁层-电离层系统对以上 超强太阳风暴的响应, 重点关注磁层大尺度结构、1 区场向电流和电离层的越极电位等空间环境要素的 变化.

PPMLR-MHD 模型采用的数值格式具有三阶空 间精度和二阶时间精度, 而且数值耗散很小, 可以在 两个计算格点内捕捉到激波或间断面。我们采用空 间直角坐标系，坐标原点选在地球中心， $x$ 轴指向太 阳. 计算区域为 $x$ 方向 $(-300,30) \operatorname{Re}$ (地球半径), $y$ 和 $z$ 方向都为 $(0,150) R e$. 地球磁层-电离层都假设关于赤 道面和昼夜子午面对称。整个计算区域划分为 $160 \times$ 


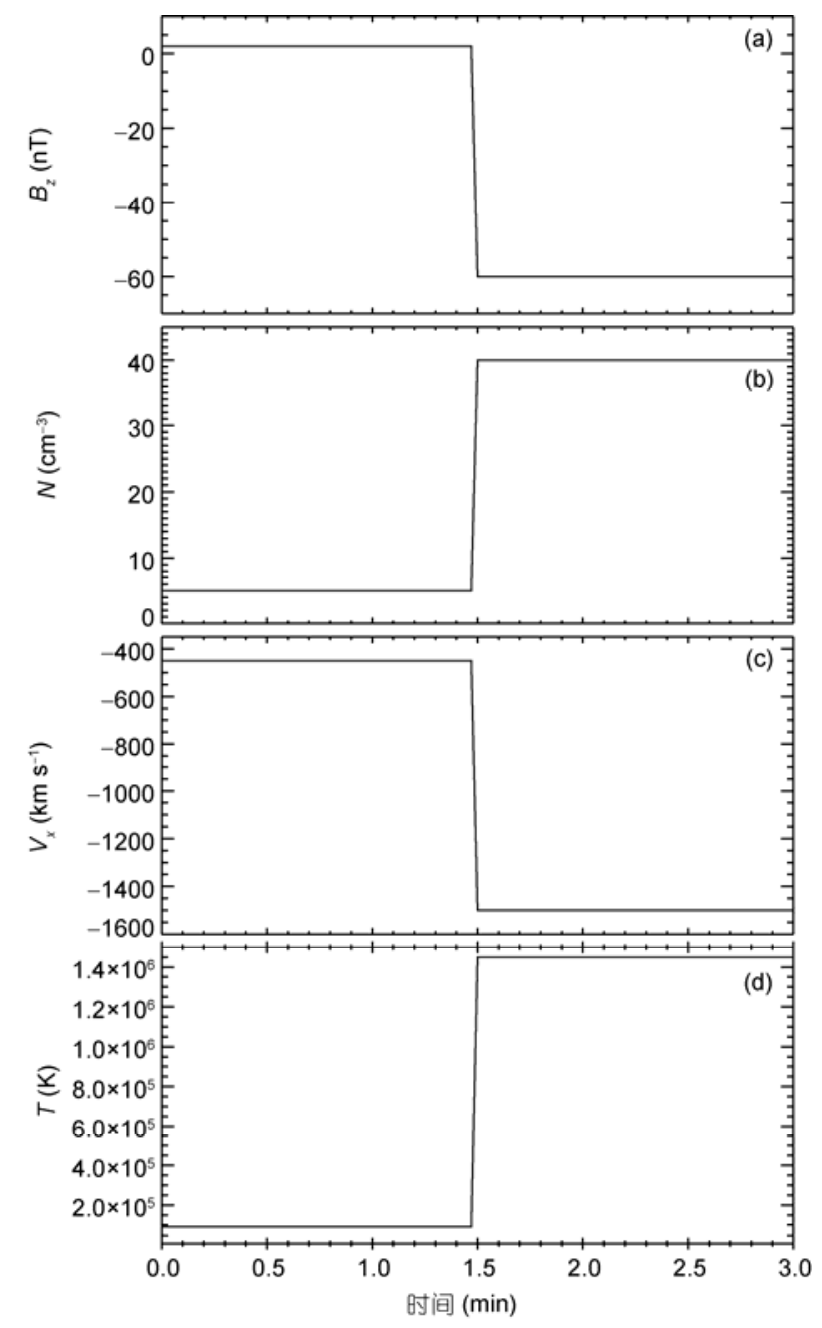

图 1 卡林顿事件的行星际条件估计

(a) 行星际磁场 $z$ 分量; (b) 太阳风密度; (c) 速度; (d) 温度

$80 \times 80$ 格点, 在 $10 \mathrm{Re}$ 之内, 格间距是均匀的 $(0.4 \mathrm{Re})$, $10 \mathrm{Re}$ 之外的格间距以 1.05 的比率增加. 计算的内边 界取在 $3 \mathrm{Re}$ 处, 内边界和电离层的耦合模型采用静 电模型, 简单起见, 本算例的电离层电导采用均匀电 导模型, Pedersen 电导取为 $15 \mathrm{~S}$, Hall 电导取为 $0 \mathrm{~S} .30$ $\operatorname{Re}$ 处的采用太阳风入流条件. $-300 \mathrm{Re}$ 处采用出流边 界条件. 计算过程分为两步: 第一步固定初始太阳风 条件, 让计算一直进行下去, 直到得到准静态的磁层电离层系统; 第二步将图 1 所示的暴时太阳风条件引 入到 $30 \mathrm{Re}$ 的入流边界.

图 2 给出了模型计算的昼夜子午面磁层大尺度 结构, 图中颜色表示的是等离子体速度 $x$-分量 $(V x)$ 的 分布. 图 2(a)为超强太阳风暴影响之前的背景太阳风
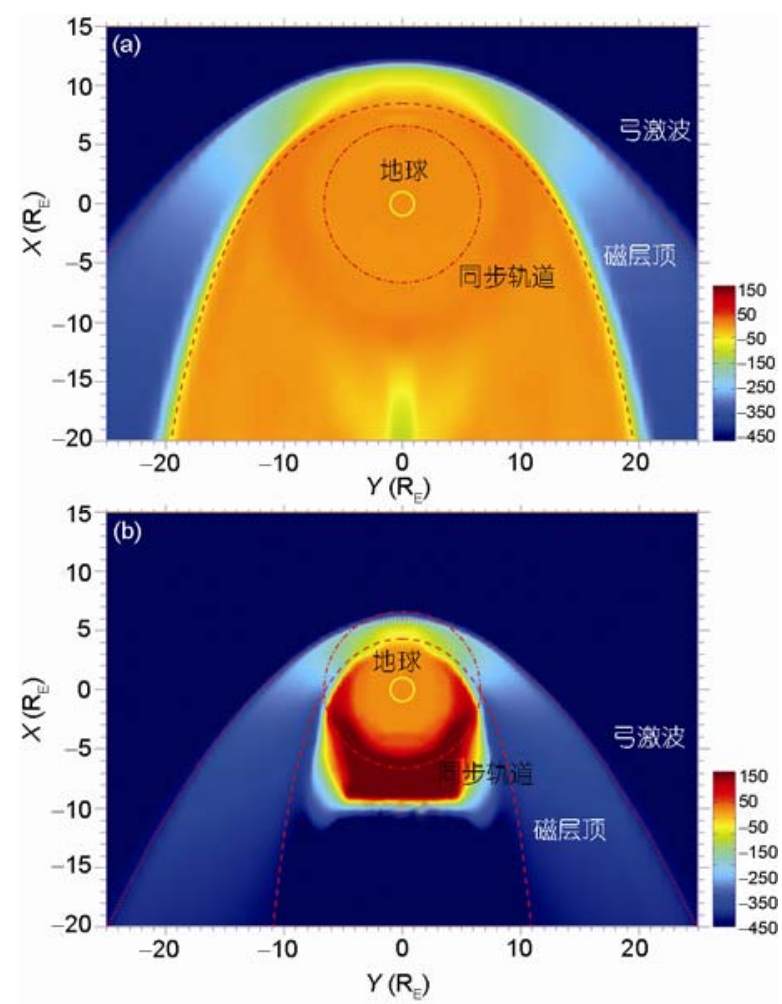

图 2 昼夜子午面内磁层大尺度结构

等离子体速度 $x$ 分量 $(V x)$ 的等值线. (a) 典型行星际条件下; (b) 超强 太阳风暴影响约 4 分钟后

条件下的典型磁层结构, 图中黄色圆圈表示地球, 点 化线的圆圈是同步轨道的位置, 再往外的红色虚线 和点线分别为磁层顶和弓激波的拟合曲线. 磁层顶 和弓激波与日地连线 ( $x$ 轴) 分别交于 8.5 和 $12.0 \mathrm{Re}$, 与 Shue98 的经验公式计算的结果一致 ${ }^{[7]}$.

图 2(b)为引入超强太阳风暴的条件约 4 分钟后的 磁层结构. 由于行星际太阳风动压急剧增大, 且行星 际磁场转强南向, 磁层顶在受到强大动压压缩的同 时, 向阳侧磁层顶处的地磁场也受到强烈的剥蚀作 用, 磁层顶和弓激波与日地连线的交点地向收缩为 4.3 和 $6.0 \mathrm{Re}$, 向阳侧磁层顶和弓激波均已缩至地球 同步轨道(距地心约 $6.6 \mathrm{Re}$ )内. $\mathrm{Li}^{\text {等 }}{ }^{[16]}$ 对 211 个磁暴 事件时的统计分析表明，中小磁暴时 $(-100<D s t \leqslant-50$ $\mathrm{nT}$ ), 同步轨道磁场的全景特征为钟形正态分布, 正 午侧 $B_{z}$ 最大, 向午夜侧减小, 而超强磁暴时 $(D s t \leqslant$ -300 nT)此分布反向, 这主要是由于超强磁暴时向阳 侧磁层顶被压缩、剥蚀到地球同步轨道以内引起的. 在强暴期间, 磁层顶压缩到地球同步轨道内是普遍 
现象, 地球同步轨道卫星直接暴露在太阳风中, 同步 卫星由于缺少地球磁层的保护而更容易受到太阳宇 宙线的损害. 同时, 侧翼部分的弓激波和磁层顶也受 到强烈压缩作用. 弓激波与 $y$ 轴和 $z$ 轴的交点分别从 $21.5 \mathrm{Re}$ 压缩至 $12 \mathrm{Re}$ 左右. 磁层顶与 $y$ 轴和 $z$ 轴的交 点分别从 13.0 和 $13.5 \mathrm{Re}$ 压缩至 6.5 和 $7.7 \mathrm{Re}$. 对于 两侧的压缩, Shue98 的经验公式给出的值与数值模拟 的值不符合, 例如, 根据经验公式计算得到磁层顶与 $y$ 轴的交点为 $9.1 \mathrm{Re}$, 明显高于模拟计算的结果 6.5 Re. 由于经验公式根据的强磁暴事件的样本有限, 在极端太阳风条件下给出的磁层顶位置和形状不能 完全反映实际情况.

超强太阳风暴的强烈压缩将导致磁层顶面电流 的急剧增大以抗衡极端太阳风条件下的压力作用, 引起电离层电流也极大增强, 发生一系列极光活动 现象, 严重时会向低纬度地区扩展 ${ }^{[17]}$. 本算例的结果 表明, 电离层 1 区场向电流在引入极端太阳风条件前 的电流密度最大为 $0.15 \mu \mathrm{A} \mathrm{m}^{-2}$, 电流总量为 $0.22 \mathrm{MA}$; 超强太阳风暴发生后, 电流急剧增强, 电流密度最大 值和电流总量分别达到了 $7.43 \mu \mathrm{A} \mathrm{m}^{-2}$ 和 $13.08 \mathrm{MA}, 1$ 区场向电流增加约 60 倍. 极光将向低纬地区扩展. 由于极端行星际磁场强南向和高速太阳风速作用下, 1 区场向电流迅速增长, 而磁层顶电流增加的幅度不 足以支持 1 区场向电流，弓激波电流将与 1 区场向电 流构成回路, 成为 1 区场向电流的 “供电源”，据估 算此时 1 区场向电流有超过 $50 \%$ 来自弓激波, 在极端 的太阳风条件下, 太阳风-磁层-电离层系统将直接通 过这种电流体系将电磁能量从行星际输入至电离层.

磁重联是太阳风与磁层耦合的主要途径, 重联 速率由沿重联线的切向电场定量描述, 沿重联线的 总电势降(重联电压) 就是总重联率. 理想磁流体力学 观点认为磁力线是等势线, 因此电离层的越极电位 等于重联电压, 从而被一些作者直接用来量度重联 ${ }^{\text {率 }}{ }^{[18]}$. 本算例中, 电离层的越极电位降从 $10.6 \mathrm{kV}$ 升 至 $868.2 \mathrm{kV}$, 增加超过 80 倍, 焦耳加热增加将超过 480 倍. Siscoe 等 ${ }^{[19]}$ 对 Hill 等 ${ }^{[20,21]}$ 的越极电位模型进 行了扩展, 得到了越极电位降与太阳风参数以及电 离层 Pedersen 电导的关系, 同时指出越极电位降的 饱和水平与太阳风动压正相关, 而与电离层 Pedersen 电导负相关. 假定该超级磁暴期间电离层 Pedersen
电导为 $15 \mathrm{~S}$, 将第 1 节的太阳风条件代入后计算得到 越极电位降为 $860.8 \mathrm{kV}$, 与我们的数值模拟结果一致. 伴随卡林顿事件有强烈的太阳质子爆发, 极区电离 层会直接受到太阳耀斑效应(SPE)的影响, 从而影响 电离层的电导率的分布和大小, 由于本文重点关注 磁层大尺度结构和变化, 为简单起见, 没有考虑电离 层精细结构的影响. 由于该事件的太阳风条件很极 端, 使得该事件越极电位降的饱和水平(约 $2000 \mathrm{kV}$ ) 远高于一般磁暴活动时期的值(约 150 250 kV). 模拟 结果表明卡林顿事件的重联电压为一般磁暴时期的 5 6 倍, 使得大量的太阳风能量进入到磁层内部, 从 而引发强烈的空间天气现象. 在磁尾区域, 由于强南 向行星际磁场作用, 在磁尾中性片区域发生磁场重 联, 由图 2(b) 可见, 在 $x=-9 \operatorname{Re}$ 附近出现反向高速流, 即为磁重联发生后的出流区域, 其中地向的高速流 将携带大量的太阳风能量进入内磁层区域, 从而影 响内磁层区域的对流结构以及相关的电离层-磁层耦 合区域的电动力学过程, 进一步影响到电离层和地 磁活动.

\section{3 讨论与结论}

1859 年发生的卡林顿事件发生时, 人类还不像 当今社会如此依赖于天基和地基的高技术系统. 当 今社会, 太阳活动导致的地球空间环境的剧烈变化, 会影响航天、通信、导航、定位和电力等领域. 随着 第 24 周太阳活动峰年(2012 2014)的临近, 超强太阳 风暴可能导致的地球空间环境的变化日益受到人们 的关注. 超强太阳风暴是低概率、高风险的空间灾害, 有关地球空间环境要素的经验公式, 由于统计样本 中超强磁暴的事例稀少, 往往不适用于如此极端条 件. 本文从 1859 年发生的卡林顿事件的地磁观测数 据出发, 估算了当时的行星际太阳风条件, 并以此为 输入, 采用全球三维磁流体力学模型定量分析了地 球磁层-电离层系统对超强太阳风暴的响应, 有关磁 层大尺度结构, 1 区场向电流和电离层越极电位降等 空间环境要素在强磁暴发生前后的值见表 1. 主要的 结论有: (1) 向阳侧磁层顶和弓激波被压缩至 4.3 和 $6.0 \operatorname{Re}$ (地球半径), 同时侧翼部分的弓激波和磁层顶 也受到强烈压缩作用. 磁层顶压缩到地球同步轨道 内是普遍现象, 地球同步轨道卫星直接暴露在磁鞘 
表 1 超强磁暴前后磁层和电离层系统空间环境要素变化

\begin{tabular}{|c|c|c|c|}
\hline & & 超强磁暴前 & 超强磁暴后 \\
\hline \multirow{3}{*}{ 磁层顶位置(Re) } & $X_{\mathrm{M}}$ & 8.5 & 4.3 \\
\hline & $Y_{\mathrm{M}}$ & 13.0 & 6.5 \\
\hline & $Z_{\mathrm{M}}$ & 13.5 & 7.7 \\
\hline \multirow{3}{*}{ 弓激波位置(Re) } & $X_{\mathrm{M}}$ & 12.0 & 6.0 \\
\hline & $Y_{\mathrm{M}}$ & 21.5 & 12.0 \\
\hline & $Z_{\mathrm{M}}$ & 21.5 & 12.5 \\
\hline \multirow{2}{*}{1 区场向电流 } & 最大电流密度 $\left(\mu \mathrm{A} \mathrm{m}^{-2}\right)$ & 0.15 & 7.43 \\
\hline & 电流总量(MA) & 0.22 & 13.08 \\
\hline & 降(kV) & 10.6 & 868.2 \\
\hline
\end{tabular}

太阳风中. (2) 超强磁暴发生后, 1 区场向电流急剧增 强约 60 倍, 电离层的越极电位降增加约 80 倍, 重联
电压为一般磁暴时期的 5 6 倍, 大量的太阳风能量 进入到磁层内部，从而引发强烈的空间天气现象.

致谢魏奉思院士、胡友秋教授和国家自然科学基金委空间天气创新研究群体成员对本项工作给予关心和指导, 国 家重大科技基础设施子午工程提供了高性能计算条件，审稿人提出修改建议，在此一并致谢.

\section{参考文献}

1 Cliver E W, Svalgaard L. The 1859 solar-terrestrial disturbance and the current limits of extreme space weather activity. Solar Phys, 2004, 224: 407-422

2 Clauer C R, Siscoe G. The great historical geomagnetic storm of 1859: A modern look. Adv Space Res, 2006, 38: 117-118

3 Green J L, Boardsen S. Duration and extent of the great auroral storm of 1859. Adv Space Res, 2006, 38: 130-135

4 Odenwald S, Green J, Taylor W. Forecasting the impact of an 1859-calibre superstorm on satellite resources. Adv Space Res, 2006, 38: 280-297

5 Shue J H, Chao J K, Fu H C, et al. A new functional form to study the solar wind control of magnetopause size and location. J Geophys Res, 1997, 102: 9497-9511

6 Lin R L, Zhang X X, Liu S Q, et al. A three-dimensional asymmetric magnetopause model. J Geophys Res, 2010, 115, doi: 10.1029/2009JA014235

7 Shue J H, Song P, Russell C T, et al. Magnetopause location under extreme solar wind conditions. J Geophys Res, 1998, 103: 17691-17700

8 Guo X C, Wang C, Hu Y Q, et al. Bow shock contributions to region 1 field-aligned current: A new result from global MHD simulations. Geophys Res Lett, 2008, 35: L03108, doi: 10.1029/2007GL032713

9 Ridley A J, De Zeeuw D L, Manchester W B, et al. The magnetospheric and ionospheric response to a very strong interplanetary shock and coronal mass ejection. Adv Space Res, 2006, 38: 263-272

10 Powell K, Roe P, Linde T, et al. A solution-adaptive upwind scheme for ideal magnetohydrodynamics. J Comput Phys, 1999, 154: 284-309

11 Tsurutani B T, Gonzalez W D, Lakhina G S. The extreme magnetic storm of September 1-2, 1859. J Geophys Res, 2003, doi: 10.1029/2002JA009504

12 Li X L, Temerin M, Tsurutani B T, et al. Modeling of 1-2 September 1859 super magnetic storm. Adv Space Res, 2006, 38: 273-279

13 Temerin M, Li X. A new model for the prediction of Dst on the basis of the solar wind. J Geophys Res, 2002, 107: 1472

14 Wang C, Li H, Richardson J D, et al. Interplanetary shock characteristics and associated geosynchronous magnetic field variations deduced from sudden impulses observed on the ground. J Geophys Res, 2010, 115, doi: 10.1029/2009JA014833

15 Hu Y Q, Guo X C, Wang C. On the ionospheric and reconnection potentials of the earth: Results from global MHD simulations. J Geophys Res, 2007, 112, doi: 10.1029/2006JA012145

16 Li H, Wang C, Kan J R. Mid-day magnetopause shifts earthward of geosynchronous orbit during geomagnetic superstorms with $D s t<-300$ nT. J Geophys Res, 2010, 115: A08230, doi: 10.1029/2009JA014612

17 Liou K, Wu C C, Lepping R P, et al. Midday sub-auroral patches (MSPs) associated with interplanetary shocks. Geophys Res Lett, 2002, 29: $16-20$ 
18 Fedder J A, Lyon J G, Slinker S P, et al. Topological structure of the magnetotail as a function of interplanetary magnetic field direction. J Geophys Res, 1995, 100: 3613

19 Siscoe G L, Erickson G M, Sonnerup B U O, et al. Hill model of transpolar potential saturation: Comparisons with MHD simulations. J Geophys Res, 2002, 107, doi: 10.1029/2001JA000109.

20 Hill T W, Dessler A J, Wolf R A. Mercury and Mars: The role of ionospheric conductivity in the acceleration of magnetospheric particles. Geophys Res Lett, 1976, 3: 429-432

21 Hill T W. Magnetic coupling between solar wind and magnetosphere: Regulated by ionospheric conductance? Eos Trans AGU, 1984, 65: 1047 